\title{
CEstrogènes et contrôle de la prolifération germinale
}

\author{
Patrick FENICHEL
}

Service d'Endocrinologie et Médecine de la Reproduction, INSERM EMI 00-09, Nice

\section{RESUME}

L'examen des souris invalidées pour les gènes de l'aromatase ou des récepteurs des estrogènes, les données cliniques d'insuffisance estrogénique primitive chez l'homme par mutation de ces mêmes gènes, les conséquences de l'exposition pré-natale au distilbène*, un estrogène puissant prescrit aux femmes enceintes entre les années 50 et 70 , et les données récentes sur les mécanismes d'action des estrogènes, invitent à réévaluer totalement le rôle des hormones stéroïdes dites "féminines" dans la physiologie de la reproduction masculine. II apparaît ainsi vraisemblable qu'une balance androgènes/estrogène appropriée soit nécessaire à la fois au cours de la différentiation sexuelle, à la descente testiculaire, à la préparation périnatale de la spermatogenèse et à son plein développement à l'âge adulte, ainsi qu'à la maturation des spermatozoïdes d'autant que le testicule exprime à la fois l'aromatase et les récepteurs RE $\alpha$ et RE $\beta$. Ces différents éléments rendent plausibles l'hypothèse du rôle des perturbateurs endocriniens environnementaux de type xénoestrogènes dans la baisse de la fertilité masculine, l'augmentation des malformations des organes génitaux et du cancer du testicule, et justifient les essais de validation scientifique. Mais les mécanismes d'action précis des estrogènes dans la physiologie de la reproduction masculine et ses perturbations restent encore très imparfaitement caractérisés.

Mots clefs : estrogènes, spermatogenèse, récepteurs des estrogènes, prolifération germinale, aromatase, cellule de Sertoli

\section{INTRODUCTION}

L'examen des souris invalidées pour les gènes de l'aromatase ou des récepteurs des estrogènes [8], les données cliniques d'insuffisance estrogénique primitive chez l'homme par mutation de ces mêmes gènes $[3,12]$, les conséquences de l'exposition pré-natale au distilbène*, un estrogène puissant prescrit aux femmes enceintes entre les années 50 et 70 [18], et les données récentes sur les mécanismes d'action des estrogènes [21], invitent à réévaluer totalement le rôle des hormones stéroïdes dites "féminines" dans la physiologie de la reproduction masculine $[13,17]$. Il apparaît ainsi vraisemblable qu'une balance androgènes/estrogène appropriée soit nécessaire au cours de la différentiation sexuelle, de la descente testiculaire, de la préparation périnatale de la spermatogenèse et de son plein développement à l'âge adulte ainsi qu'au cours de la maturation des spermatozoïdes [36], d'autant que le testicule exprime à la fois l'aromatase [6] et les récepteurs $\operatorname{RE} \alpha$ et $\operatorname{Re} \beta[11,30,42]$. Ces différents éléments rendent plausibles l'hypothèse du rôle des perturbateurs endocriniens environnementaux de type xénoestrogènes dans la baisse de la fertilité masculine, l'augmentation des malformations des organes génitaux et du cancer du testicule $[4,14,46]$ et justifient les essais de validation scientifique. Mais les mécanismes d'action précis des estrogènes dans la physiologie de la reproduction masculine et ses perturbations, restent encore très imparfaitement caractérisés.

Nous nous attacherons ici à rapporter les arguments en faveur d'un rôle direct des estrogènes sur les cellules germinales au cours de la spermatogenèse ainsi que l'estro-

\section{Correspondance}

Pr. Patrick Fénichel - Service d'Endocrinologie et Reproduction, Hôpital de L'Archet, BP 3079, 06202 Nice Cedex 3 - Email fenichel.p@chu-nice.fr 
gèno-dépendance vraisemblable des cellules germinales malignes dans le cadre du séminome, cancer testiculaire le plus fréquent.

\section{POINTS D'IMPACT POSSIBLES DES ESTROGENES SUR LA FERTILITE}

La répartition tissulaire et cellulaire de l'expression de l'activité enzymatique aromatase et des récepteurs des estrogènes RE $\alpha$ et RE $\beta$ dans l'appareil de reproduction chez I'animal et chez l'homme, par ailleurs largement développée dans cette revue, laisse envisager plusieurs points d'impact possible des estrogènes dans le contrôle de la mise en route et du maintien d'une spermatogenèse satisfaisante. Les observations faites sur les souris transgéniques ou chez l'homme sont susceptibles de fournir des éléments d'orientation. Ainsi les modèles cliniques d'insuffisance estrogénique ont démontré formellement que les gonadotrophines, en particulier la $\mathrm{LH}$, sont régulées chez l'homme par les estrogènes et non par les androgènes. En effet, en cas de mutation du gène de l'aromatase, seul l'estradiol et non la testostérone s'est avéré capable de normaliser l'élévation de la LH [9] ce qui avait déjà été suggéré par l'utilisation de testostérone et d'un inhibiteur de l'aromatase [15].

Les souris invalidées pour le gène de l'aromatase (ArKO) présentent à l'âge adulte une hyperplasie/hypertrophie des cellules de Leydig qui est vraisemblablement la conséquence d'un taux élevé de LH [40]. Néanmoins diverses expériences chez le rongeur soumis à une exposition aux estrogènes ou aux xénoestrogènes suggèrent que les estrogènes régulent de façon négative la prolifération néo-natale et pubertaire des cellules de Leydig et exercent un rétrocontrôle négatif paracrine sur la stéroïdogenèse leydigienne conduisant à la synthèse d'androgènes, principale fonction de ces cellules, indispensables à la spermatogenèse. Cet effet pourrait être médié via un mode d'action non génomique [36].

Les cellules de Sertoli prolifèrent au cours de la période fotale et péri-natale et cette prolifération, qui cède ensuite la place à une différentiation cellulaire, est classiquement considérée comme déterminante pour la future spermatogenèse. La cellule de Sertoli de rat immature exprime une activité aromatase FSH-dépendante [10]. D'autre part cette cellule exprime des récepteurs RE $\beta$ [5]. Différents arguments et l'analogie avec la cellule de la granulosa (revue dans [36]) suggèrent que les estrogènes produits par la cellule de Sertoli sous l'effet de la FSH participent, via la synthèse de TGF $\beta$ à la prolifération cellulaire mais par contre exercent ensuite un effet inhibiteur sur la maturation de ces cellules. Cette inhibition est illustrée par Sharpe et coll. [43], qui étudient les effets de l'exposition néonatale au DES et aux antagonistes de la LHRH chez le rat, et qui retrouvent à l'âge adulte des anomalies permanentes de l'histologique testiculaire et de la spermatogenèse $[1,2]$. Cette hypothèse est également renforcée par l'observation de véritables cellules de Sertoli différentiées retrouvées dans les ovaires des souris ERßKO et ArKO (revue dans [36]). Par ailleurs les estrogènes participent, en la potentialisant, à la régulation par la FSH de l'expression de la Ncadhérine dans la cellule de Sertoli immature de souris [28] et pourraient ainsi entre autre, via cette molécule d'adhésion utile aux relations germinales/Sertoli, participer à la structuration de l'épithélium séminifère [38].

Les souris mâles ER $\alpha$ KO sont stériles et présentent une dilatation des tubes séminifères avec destruction de la spermatogenèse [20]. Cet effet est lié à la non résorption du fluide testiculaire au niveau des tubes efférents entraînant une dilatation des cavités sus-jacentes [16]. Cette résorption physiologique est sous la dépendance des estrogènes, via leurs récepteurs $\mathrm{ER} \alpha$, qui régulent l'expression de l'aquaporine-1 qui contrôle les mouvements d'eau à travers l'épithélium canalaire [20]. Par ailleurs la fonction épididymaire qui est régulée par les androgènes pourrait être également sous le contrôle des estrogènes pour lesquels il existe des récepteurs au niveau de l'épithélium [5].

\section{EFFETS DIRECTS DES ESTROGENES SUR LES CELLULES GERMINALES}

La spermatogenèse implique une phase de prolifération concernant les spermatogonies, une phase de maturation méiotique des spermatocytes I, une phase de différentiation touchant les spermatides. Ces différentes étapes au sein du tube séminifère sont sous le contrôle endocrinien de la FSH et de la testostérone via la cellule de Sertoli. Il a été récemment suggéré que les estrogènes étaient également impliqués dans ces étapes. Les spermatozoïdes vont ensuite subir une maturation au cours de leur transit dans les voies génitales masculines et féminines dont les fluides comprennent des estrogènes [25].

\section{La prolifération des cellules germinales souches}

Les estrogènes pourraient jouer un rôle anti-apopototique sur les cellules germinales souches et favoriser ainsi la prolifération des gonocytes en période péri-natale [24, 39] et des spermatogonies à l'âge adulte [31]. En effet, de faibles doses d'estradiol $(1 \mu \mathrm{M})$ mais non de plus fortes, sont capables de stimuler la prolifération de gonocytes de rat, et cet effet est inhibé par un antagoniste des récepteurs des estrogènes ICI 164364 [24]. De faibles doses d'E2 comme de fortes doses de dihydrotestosterone sont capables de prévenir l'apoptose de spermatocytes et de spermatides humaines au sein de tubes séminifères cultivés in vitro [37]. La rapidité de l'effet, constaté en environ 4 heures, suggère un effet non génomique. Aussi bien les gonocytes 
que les spermatogonies expriment une activité aromatase et des récepteurs $\beta$, une seule équipe ayant retrouvé des récepteurs $\alpha$ dans certaines cellules germinales humaines [37]. La source d'estrogène pourrait également être sertolienne.

\section{La spermiation}

Les souris ArKo présentent à l'âge adulte au sein des tubes séminifères une apoptose exagérée des spermatides rondes [40]. En effet, dès 18 semaines le nombre de spermatides rondes et allongées diminue de façon significative alors que les spermatogonies et les spermatocytes sont inchangés [40]. Le rôle des estrogènes sur la spermiation est également suggéré par la réduction du nombre de spermatides allongées chez le singe adulte traité par un inhibiteur de l'aromatase [44]. Les spermatides rondes des souris ArKo présentent également de façon surprenante des anomalies de l'acrosome qui laissent supposer que ce processus de vésiculisation à partir de l'appareil de Golgi est estrogénodépendant d'autant que le golgi des spermatides contient de l'aromatase [35] et que les spermatides expriment RE $\beta$ [5].

\section{La maturation des spermatozoïdes}

De très anciens travaux avaient mis en évidence l'activation des spermatozoïdes par les estrogènes via une action rapide membranaire dite non génomique [7]. Très récemment a été décrit un récepteur des estrogènes à la surface du spermatozoïde, reconnu par un anticorps anti RE $\alpha$ d'un poids moléculaire de $29 \mathrm{kDa}[26,27]$, et impliquant une voie de signalisation via l'AMPc, l'entrée rapide de calcium et/ou la voie des MAPkinases. Les estrogènes sont présents à des taux élevés dans les voies excrétrices masculines et le taux séminal est supérieur au taux sanguin [25]. Les estrogènes et la progestérone sont, bien entendu, présents dans les fluides des voies génitales féminines.

\section{LES SOURIS ET LES HOMMES AVEC INSUFFISANCE ESTROGENIQUE SONT-ILS STERILES ?}

Il semble bien tout d'abord que les troubles de la spermatogenèse chez les souris mâles ER $\alpha$ KO soient la conséquence en amont d'un problème de non résorption du fluide au niveau des canaux efférents et non d'un problème intrinsèque aux cellules germinales, car celles-ci sont capables de se développer lorsqu'elles sont transplantées chez la souris sauvage [29]. Ce qui est logique, car il n'y a pas d'expression de RE $\alpha$ dans les cellules germinales normales [42]. Par contre celles-ci expriment ER $\beta$ et il est donc plus difficile de comprendre pourquoi jusqu'à présent il n'a pas été constaté de troubles de la fertilité chez les souris mâles $\mathrm{ER} \alpha \mathrm{KO}$ [22]. Une des explications pourrait être une com- pensation via $E R \alpha$ et/ou l'intervention des voies non génomiques que nous venons d'évoquer via des récepteurs des estrogènes associés aux membranes [21, 26]. Quant aux souris ArKO, plusieurs lignées transgéniques ont été obtenues à partir de laboratoires différents montrant toutes à 14 semaines une spermatogenèse normale ; pour l'une d'entre elles il existe une altération progressive de la spermatogenèse complète à un an [40], pour une autre il y a diminution de l'épaisseur des tubes séminifères et pour la troisième l'histologie adulte manque. Il est difficile de comprendre ces différences de phénotype, mais il est possible que l'exposition in utero aux estrogènes maternels et les phytoestrogènes présents dans la nourriture de la mère allaitante ou donnés directement puissent contribuer à la variabilité des phénotypes [36].

En ce qui concerne les hommes atteints d'une mutation du gène de l'aromatase, l'un n'a pas eu de spermogramme et a présenté une puberté normale avec une macroorchidie liée à l'élévation de la LH [33] ; un autre présentait une oligoasthénospermie avec, à la biopsie testiculaire, une hypospermatogenèse avec arrêt de la maturation mais il n'est pas impossible qu'il existait chez lui une cause familiale associée d'hypofertilité [3]. Enfin le troisième cas décrit récemment [32] présentait des antécédents d'ectopie testiculaire bilatérale et un spermogramme altéré avec asthénospermie. Quant au sujet présentant une mutation de RE $\alpha$ [45], une macroorchidie a également été notée chez lui, ainsi qu'une numération normale au spermogramme mais avec une viabilité très diminuée des spermatozoïdes.

\section{ESTROGENO-DEPENDANCE DES CEL- LULES SEMINOMATEUSES}

Le cancer du testicule, le cancer le plus fréquent de l'homme jeune, semble depuis quelques décennies en constante augmentation. Les constatations épidémiologiques, faites dans les mêmes régions géographiques, mettant en évidence une baisse des paramètres du spermogramme, et l'élévation des anomalies du tractus génital masculin (cryptorchidie, hypospadias), ont conduit à suggérer la responsabilité éventuelle de polluants industriels de type pesticides à activité hormonale estrogénique ou anti-androgénique [14, 34]. Des arguments expérimentaux chez le rongeur [19] ou les constatations faites chez les hommes dont les mères ont été traitées par le distilbène [18] sont également à prendre en considération. Le séminome, ou prolifération anarchique et maligne de cellules germinales, constitue de loin le cancer testiculaire le plus fréquent (90\% des cas) seul ou associé à une autre forme histologique. Il pourrait dériver de gonocytes, dont il partage le marqueur de la phosphatase alcaline placentaire, qui ne se seraient pas différentier en spermatogonies dans la période péri-natale.

Dans notre laboratoire, nous avons mis en évidence dans 
les séminomes l'expression du gène et de la protéine du récepteur RE $\beta$ et non RE $\alpha$ [41]. Grâce à la collaboration de Serge Carreau, il a été possible également de mettre en évidence l'ARN messager de l'aromatase suggérant que les cellules séminomateuses sont capables de synthétiser des estrogènes à partir des androgènes et de répondre aux estrogènes [41]. En effet, en utilisant une lignée séminomateuse humaine nous avons constaté une double action des estrogènes sur la prolifération des cellules séminomateuses. Il existe d'abord une action stimulatrice précoce à point de départ membranaire, car induite par E2 couplé à la BSA, qui emprunte la voie de signalisation des MAP-kinases et qui ne semble pas médiée par RE $\beta$ car non inhibée par un antagoniste des récepteurs des estrogènes ICI 182780 [41]. Il existe également une action inhibant la prolifération cellulaire, action prolongée et prédominante qui pourrait quant à elle impliquée $\operatorname{RE} \beta$ car antagonisée par ICI 182780 [41]. Les voies de signalisation précises utilisées par les estrogènes pour contrôler la prolifération séminomateuse sont actuellement en cours d'étude au laboratoire. Il est trop prématuré d'en tirer pour l'instant des conséquences pour la prolifération des cellules germinales normales et pathologiques. Mais ceci évoque une éventuelle estrogèno-dépendance du cancer du testicule de type séminome.

\section{CONCLUSION}

Ainsi il existe aujourd'hui des arguments nombreux expérimentaux et cliniques qui suggèrent que les estrogènes jouent un rôle non négligeable associés à la FSH et à la testostérone dans le contrôle de la spermatogenèse. Leur source et leur action peuvent être indirectes via la cellule de Sertoli, mais ils peuvent aussi intervenir également directement au niveau des cellules germinales capables d'exprimer l'aromatase et des récepteurs aux estrogènes. Plusieurs voies de signalisation sont susceptibles d'être mises en jeu par les estrogènes. Néanmoins il reste à préciser l'importance exacte des estrogènes au cours de la spermatogenèse normale et pathologique.

\section{REFERENCES}

1. ATANASSOVA N., MCKINNEL C., TURNER K.J, et al. : Comparative effects of neonatal exposure of male rats to potent and weak (environnemental) estrogens on spermatogenesis at puberty and the relationship to adult testis size and fertility : evidence for stimulatory effects of low estrogen levels. Endocrinology, $2000,141: 3898-3907$.

2. ATANASSOVA N., MCKINNEL C., TURNER K.J. et al. : Permanent effects of neonatal estrogen exposure in rats on reproductive hormone levels, Sertoli cell number, and the efficiency of spermatogenesis in adulthood. Endocrinology, 1999, 140 : 5364-5373.
3. CARANI C., QIN K., SIMONI M., et al. : Effect of testosterone and estradiol in a man with aromatase deficiency. N. Engl. J. Med., 1997, 337 : 91-95.

4. CARLSEN E., GIWERCMAN A., KEIDING N., SKAKKEBAECK N. : Evidence for decreasing quality of semen during past 50 years. Brit. Med. J., 1992, 305 : 609-613.

5. CARREAU S. : Estrogènes et fonctions testiculaires. Reprod. Hum. Hormon., 2000, $13: 1-10$.

6. CARREAU S., BOURGUIBA S., GALERAUD-DENIS I. et al. : Aromatase expression in male germ cells. J. Steroid. Biochem. Mol. Biol. 2001, 79 : 203-208.

7. CHENG C.Y., BOETTCHER B. : The effects of steroids on the in vitro migration of washed human spermatozoa in modified tyrode's solution or in fasting human blood serum. Fertil. Steril., 1979, 32 : 566-570.

8. COUSE J.F., KORACH K.S. : Estrogen receptor null mice : what have we learned and where will they lead us ? Endocr. Rev., 1999, $20: 358-417$.

9. DELADOEY J., FLÜCK C., BEX M., YOSHIMURA N., HARADA N., MULLIS P. : Aromatase deficiency caused by a novel P450arom gene mutation : impact of absent estrogen production on serum gonadotropin concentration in a boy. J. Clin. Endocrinol. Metab., 1999, 84 : 4050-4054.

10. DORRINGTON J.H., BENDELL J.J., KHAN S.A. : Interactions between FSH, estradiol-17 $\beta$ and transforming growth factorregulate growth and differentiation in the rat gonad. J. Steroid. Biochem. Mol. Biol., 1993, 44 : 441-447.

11. ENMARK E., PELTO-HUIKKO M., GRANDDIEN K. et al. : Human estrogen receptor ßgene structure, chromosomal localization, and expression pattern. J. Clin. Endocrinol. Metab., 1997, $82: 4258-4265$.

12. FAUSTINI-FUSTINI M., ROCHIRA V., CARANI C. : Oestrogen deficiency in men : where are we today? Eur. J. Endocrinol., 1999, $140: 111-129$.

13. FENICHEL P. et al. : Mises au point cliniques d'endocrinologie. In : N. Guétitée et $\mathbf{J}$. Leclère eds. Nutrition et Métabolisme, Paris, 2002 : 133-141.

14. FENICHEL P. : Le point sur hormones et imposteurs endocriniens dans l'eau et la chaîne alimentaire : impact possible en clinique et reproduction humaine. Comte Rendu du Workshop de Risghospitalet : Copenhagen 27-30 Mai 2000. pp20. Ipsen Biotech.

15. FINKELSTEIN J.S., WHITCOMB R.W., O'DEA L.S., LONSCOPE C., SHOENFELD D.A., CROWLEY W.F. Jr. : Sex steroid control of gonadotrophin secretion in the human male. I : Effect of testosterone administration in normal and gonadotropin-releasing hormone-deficient men. J. Clin. Endocrinol. Metab., 1991, $73: 609-620$.

16. FISHER J.S., TURNER K.J., FRASER H.M., SAUNDERS P.T.K., BROWN D., SHARPE R.M. : Immunoexpression of aquaporin-1 in the efferent ducts of the rat and marmoset monkey during development, its modulation by estrogens, and its possible role in fluid resorption. Endocrinology., 1998, 139 : 3935-3945.

17. GALERAUD-DENIS I., MARIE E., CARREAU S. : Estrogènes et spermatozoïdes. Andrologie, 1999, $9: 252-260$. 
18. GILL W.B., SCHUMACHER G.F., BIBBO M., SRAUS F.H.D., SHOENBERG H.W. : Association of diethylstilbestrol exposure in utero with cryptorchidism, testicular hypoplasia and semen abnormalities. J. Urol., 1979, 122 : 36-39.

19. GRAY L.E. Jr. : Xenoendocrine disrupters : laboratory studies on male reproductive effects. Toxicol. Lett., 1998, $102: 331-335$.

20. HESS R.A., BUNICK D., LEE K.H. et al. : A role for estrogens in the male reproductive system. Nature, 1997, $390: 509-512$.

21. KELLY M., LEVIN E.R. : Rapid actions on plasma membrane estrogen receptors. Trends Endocrinol. Metab., 2001, 12 : 152156.

22. KREJE J.H., HODGIN J.B., COUSE J.F. et al. : Generation and reproductive phenotypes of mice lacking estrogen receptor beta. Proc. Natl. Acad. Sci., 1998, 22 : 15677-15682.

23. KUIPER G., ENMARK E., PELTO-HUIKKO M., NILSSON S., GUSTAFSSON J.A. : Cloning of a novel estrogen receptor expressed in rat prostate and ovary. Proc. Natl. Acad. Sci., 1996, 93 : 5925-5930.

24. LI H., PAPADOPOULOS V., VIDIC B., et al. : Regulation of rat testis gonocyte proliferation by platelet-derived growth factor and estradiol : identification of signaling mechanisms involved. Endocrinology, 1997, $138:$ 1289-1298.

25. LUBOSHITZKY R., SHEN-ORR Z., HERER P. : Seminal plasma melatonin and gonadal steroids concentration in normal men. Arch. Androl., 2002, 48 : 225-232.

26. LUCONI M., FORTI G., BALDI E. : Genomic and nongenomic effects of estrogens : molecular mechanisms of action and clinical implications for male reproduction. J. Steroid. Biochem. Mol. Biol., 2002, 80 : 369-381.

27. LUCONI M., MURATORI M., FORTI G., BALDI E. : Identification and characterization of a novel functional estrogen receptor on human sperm membrane that interferes with progesterone effects. J. Clin. Endocrinol. Metab., 1999, 84 : 1670-1678.

28. MACCALMAN C.D., GETSIOS S., FARROOKHI R., BLASHUK O.W. : Estrogens potentiate the stimulatory effects of follicle-stimulating hormone on $\mathrm{N}$-cadherin messenger ribonucleic acid levels in cultured mouse Sertoli cells. Endocrinology, 1997, $138: 41-48$.

29. MAHATO D., GOULDING E.H., KORACH K.S., EDDY E.M. : Spermatogenic cells do not require estrogen receptor a for development or function. Endocrinology, 2000, 141 : 1273-1276.

30. MÄKINEN S., MÄKELÄ S., WEIHUA Z., et al. : Localization of estrogen receptors alpha and beta in human testis. Mol. Hum. Reprod., 2001, 7 : 497-503.

31. MIURA T., MIURA C., OHTA T., NADER M.R., TODO T., YAMAUCHI K. : Estradiol-17 $\beta$ stimulates the renewal of spermatogonial stem cells in males. Biochem. Biophys. Res. Commun., 1999, $264: 230-234$.

32. MOREAU F., PURA M., MITTRE H., REZNIK Y., CARREAU S., KOTTLER M.L. : Déficit en estrogènes par mutation inactivatrice du gène de l'aromatase chez l'homme. Ann. Endocrinol., 2002, $63: 269$.

33. MORISHIMA A., GRUMBACH M.M., SIMPSON E.R., FIS-
HER C., QIN K. : Aromatase deficiency in male and female siblings caused by a novel mutation and the physiological role of estrogens. J. Clin. Endocrinol. Metab., 1995, 80 : 3689-3690.

34. NEF S., SHIPMAN T., PARADA L.F. : A molecular basis for estrogen-induced cryptorchidism. Dev. Biol., 2000, 224 : 354 361.

35. NITTA H., BUNICK D., HESS R.A., et al. : Germ cells of the mouse testis express P450 aromatase. Endocrinology, 1993, 132: 1396-1341.

36. O'DONNELL L., ROBERTSON K.M., JONES M.E., SIMSON E.R. : Estrogen and spermatogenesis. Endocr. Rev., 2001, 22 : 289-318.

37. PENTIKAINEN V., ERKKILA K., SUOMALAINEN L., PARVINEN M., DUNKEL L. : Estradiol acts as a germ cell survival factor in the human testis in vitro. J. Clin. Endocrinol. Metab., 2000, $85: 2057-2067$.

38. PERRYMAN K.J., STANTON P.G., LOVELAND K.L., MACLACHLAN R.I., ROBERTSON D.M. : Hormonal dependency of neural cadherin in the binding of round spermatids to Sertoli cells in vitro. Endocrinology. 1996, $137: 3877-3883$.

39. LI H., PAPADOPOULOS V., VIDIC B., DYM M., CULTY M. : Regulation of rat testis gonocyte proliferation by platelet-derived growth factor and estradiol: identification of signaling mechanisms involved. Endocrinology, 1997, 138 : 1289-1298.

40. ROBERTSON K.M., O'DONNELL L., JONES M.E.E. et al. : Impairment of spermatogenesis in mice lacking a functional aromatase (cyp 19) gene Proc. Natl. Acad. Sci., 1999, 96 : 79867991.

41. ROGER C., MOGHRABI B., CHEVALLIER D. et al. : Le cancer du testicule est-il un cancer estrogèno-dépendant ? Effets des estrogènes sur la prolifération des cellules séminomateuses. Ann. Endocrinol., 2002, $63: 350$.

42. SAUNDERS P.T., SHARPE R.M., WILLIAMS K. et al. : Differential expression of estrogen receptor $\alpha$ and $\beta$ proteins in the testes and male reproductive system of human and non-human primates. Mol. Hum. Reprod., 2000, 7 : 227-236.

43. SHARPE R.M., ATANASSOVA N., MCKINNELL C. et al. : Abnormalities in functional development of the Sertoli cells in rats treated neonatally with diethylstilbestrol : a possible role for estrogens in Sertoli cell development. Biol. Reprod., 1998, 59 : 1084-1094.

44. SHETTY G., KRISHNAMURTY H., KRISHNAMURTY H.N., BHATNAGAR A.S., MOUDGAL N.R. : Effect of long term treatment with aromatase inhibitor on testicular function of adult male Bonnet monkeys (M.Radiata). Steroids, 1998, 63 : 414420.

45. SMITH E.P., BOYD J., FRANK R., TAKAHASHI H., COHEN R.M., SPEKER B. : Estrogen reistance caused by a mutation in the estrogen-receptor gene in a man. N. Engl. J. Med., 1994, 331: 1056-1061.

46. TOPPARI J., LARSEN J.C., CHRISTIANSEN P. et aI. : Male reproductive health and environmental xenoestrogens. Environ. Health Perspect., 1996, 104 : 741-803. 


\section{ABSTRACT \\ Estrogens and germ cell regulation}

\section{Patrick Fénichel}

The role of so-called female sex steroids in males needs to be completely re-evaluated in the light of the clinical features of male estrogen deficiency, new mechanisms of estrogen receptors, and description of mice lacking functional aromatase or estrogen receptor gene. An appropriate balance between estrogens and androgens appears to be necessary to allow full sexual development and reproduction. All these characteristics support the hypothesis of the role of endocrine disruptors and justify investigation of the possible role of environmental xenoestrogens on male fertility, genital malformations or cancer.

Key-words: estrogens, spermatogenesis, estrogen receptors, germ cell proliferation, aromatase, Sertoli cell 